\title{
Interpretation of second solar spectrum observations of the Sr I $4607 \AA$ line in a quiet region: Turbulent magnetic field strength determination ${ }^{\star}$
}

\author{
V. Bommier ${ }^{1}$, M. Derouich ${ }^{1}$, E. Landi Degl'Innocenti ${ }^{2}$, G. Molodij ${ }^{3}$, and S. Sahal-Bréchot ${ }^{1}$ \\ 1 Laboratoire d'Étude du Rayonnement et de la Matière en Astrophysique, CNRS UMR 8112 - LERMA, \\ Observatoire de Paris, Section de Meudon, 92195 Meudon, France \\ e-mail: v.bommier@obspm.fr \\ 2 Università degli Studi di Firenze, Dipartimento di Astronomia e Scienza dello Spazio, Largo E. Fermi 2, 50125 Firenze, Italy \\ 3 Laboratoire d'Études Spatiales et d'Instrumentation en Astrophysique, CNRS UMR 8109 - LESIA, Observatoire de Paris, \\ Section de Meudon, 92195 Meudon, France
}

Received 1 December 2003 / Accepted 23 October 2004

\begin{abstract}
This paper presents and interprets some observations of the limb polarization of Sr I $4607 \AA$ obtained with the spectropolarimeter of the French-Italian telescope THEMIS in quiet regions close to the solar North Pole on 2002 December 7-9. The linear polarization was measured for a series of limb distances ranging from 4 to $160 \mathrm{arcsec}$, corresponding to heights of optical depth unity in the line core ranging from about 330 to $220 \mathrm{~km}$, respectively, above the $\tau_{5000}=1$ level. To increase the polarimetric sensitivity, the data were averaged along the spectrograph slit (one arcmin long) set parallel to the solar limb. Since the data show no rotation of the linear polarization direction with respect to the limb direction, the observed depolarization is ascribed to the Hanle effect of a turbulent weak magnetic field, the zero-field polarization being derived from a model. The interpretation is performed by means of an algorithm which describes the process of line formation in terms of the atomic density matrix formalism, the solar atmosphere being described by an empirical, plane-parallel model. The collisional rates entering the model (inelastic collisions with electrons, elastic depolarizing collisions with neutral hydrogen), have been computed by applying fast semi-classical methods having a typical accuracy of the order of $20 \%$ or better (see Derouich 2004), leading to 6\% inaccuracy on the magnetic field strength determination. We assume a unimodal distribution for the intensity of the turbulent field. The computed intensity profile has been adjusted to the observed one in both depth and width, by varying both microturbulent and macroturbulent velocities. The best adjustment is obtained for respectively $1.87 \mathrm{~km} \mathrm{~s}^{-1}$ (micro) and $1.78 \mathrm{~km} \mathrm{~s}^{-1}$ (macro). The evaluation of the magnetic depolarization leads then to the average value of 46 Gauss for the turbulent magnetic field strength, with a gradient of $-0.12 \mathrm{Gauss} / \mathrm{km}$. Our results are in very good agreement with the value of 60 Gauss determined at large $\mu$, in the volume-filling field case, by Trujillo Bueno et al. (2004, Nature, 430, 326), using a 3D magneto-convective simulation. This validates our method.
\end{abstract}

Key words. atomic processes - line: formation - line: profiles - Sun: magnetic fields - polarization - radiative transfer

\section{Introduction}

Spectral lines are linearly polarized close to the solar limb. This phenomenon has been known for a long time and is thought to be due to the scattering by atoms of the solar radiation which is particularly anisotropic in the top layers of the solar atmosphere. The spectrum of the linear polarization, for which the name of "second solar spectrum" was suggested by V.V. Ivanov during the First Solar Polarization Workshop (St Petersburg, Russia, 8-12 May 1995), shows structures very

* based on observations made with the french-italian telescope THEMIS operated by CNRS and CNR on the island of Tenerife in the spanish Observatorio del Teide of the Instituto de Astrofísica de Canarias. different from those of the intensity spectrum, and is therefore rich in new information, particularly on symmetry-breaking features like velocity or magnetic fields. The Sr I $4607 \AA$ line was found to be one of the most polarized of the second solar spectrum (Stenflo et al. 1983a,b), so that further observations have been devoted to define its center-to-limb behavior (Stenflo et al. 1997), in particular with the spectropolarimeter of the French-Italian THÉMIS solar telescope (Faurobert et al. 2001; Trujillo Bueno et al. 2001; Bommier \& Molodij 2002; and the present observations). An atlas of the second solar spectrum was also obtained with the ZIMPOL II polarimeter at IRSOL, Locarno (Gandorfer 2000, 2002). This atlas provides a further observation of the linear polarization of Sr I $4607 \AA$ at 5 arcsec from the solar limb. 

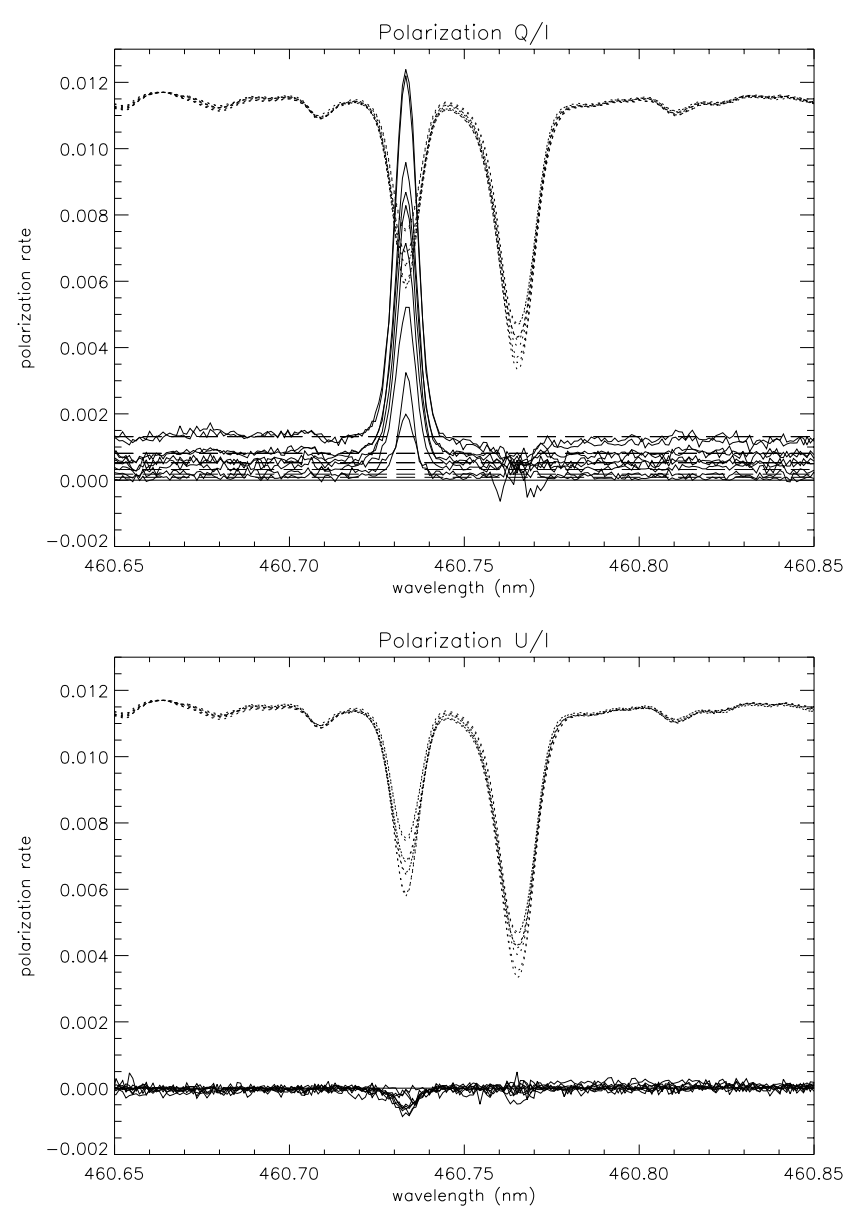

Fig. 1. Superimposed observations for all the limb distances listed in Table 1. The Stokes parameters $Q$ and $U$ are expressed in the limb reference frame. Dotted lines: intensity $I$ in arbitrary units. The slit has been positioned parallel to the solar limb and the data have been averaged along the slit (one arcmin long) to increase the polarimetric sensitivity. The Sr I line is $460.7333 \mathrm{~nm}$ and the neighboring line at $460.7651 \mathrm{~nm}$ is a Fe I line that depolarizes the continuum. The broken line is the theoretical value of the limb polarization to which the observed continuum polarization has been superimposed (see text for details).

When observing outside active regions, the linear polarization of the SrI $4607 \AA$ line, which is of the order of $1 \%$ at 5 arcsec from the solar limb, has been found to be parallel to the limb direction, as confirmed by the present observations (see Sect. 2 and Fig. 1). When observing with low spatial resolution, no rotation of the polarization direction with respect to the limb direction is observed (see also Trujillo Bueno et al. 2001; and Stenflo 2002). Such a rotation would be introduced in the scattering polarization by the presence of a deterministic, weak magnetic field (Hanle effect). These features make this line particularly suitable for attempting a theoretical interpretation of its polarization degree. Fortunately, the 2-level approximation is suitable for its interpretation because the $4607 \AA$ line is the resonance line of the SrI atom, $5 \mathrm{~s}^{2}{ }^{1} \mathrm{~S}_{0} \rightarrow 5 \mathrm{~s} 5 \mathrm{p}{ }^{1} \mathrm{P}_{1}$, with a Landé factor of the upper level $g=1$. Depolarizing effects due to hyperfine structure can be safely neglected since the only isotope of Sr having nuclear spin $I \neq 0$ has a very low natural abundance (7\%). Relatively simple polarized radiative transfer models have been built up, which show that the theoretical linear polarization degree, evaluated for a plane-parallel atmosphere in the absence of a magnetic field, is higher than the observed one. This resulting depolarization effect (joined to the fact that no rotation of the polarization direction is observed) has been ascribed to the Hanle effect due to a turbulent weak magnetic field (see the first model in Faurobert-Scholl 1993; and the most recent one developed for the interpretation of the THÉMIS data, in Faurobert et al. 2001).

To model the magnetic depolarization, the further depolarization effect due to collisions of the $\mathrm{Sr}$ atoms with neutral hydrogen atoms has to be taken into account. The corresponding rates were poorly known, but quantum chemistry calculations have been recently developed (see the first calculations and resulting interpretation in Faurobert-Scholl et al. 1995), and are now available for a better diagnostic of the turbulent magnetic field.

The aim of the present work is threefold:

- To achieve precise polarimetric observations with the THÉMIS telescope at several distances from the limb by using the beam exchange technique described in Bommier $\&$ Molodij (2002). We have obtained measurements ranging from 4 to 160 arcsec from the solar limb. The slit has been positioned parallel to the solar limb in a quiet region (North Pole), and the data have been averaged along the slit to increase polarimetric sensitivity. Our results display the linear polarization Stokes parameters, showing in particular the absence of rotation of the polarization direction (see Sect. 2 and Fig. 1).

- To apply the atomic density matrix method for polarized radiative transfer developed by Landi Degl'Innocenti et al. (1990) to model the line polarization. Although based on a different approach for solving the coupled equations of statistical equilibrium and non-LTE radiative transfer, this method is in principle equivalent to the "scattering method" used by Faurobert-Scholl (1993), and should obviously produce the same results. Complete frequency redistribution is assumed (see a more detailed description of the method in Sect. 3).

- To develop fast semi-classical methods for computing the collisional rates, validated by comparison of their results with more complex methods based on quantum chemistry. The interest of these approximate methods is their ability to evaluate collisional rates with a typical accuracy of about $20 \%$ or better, without any need for using heavier and more time-consuming quantum chemistry methods. Such a semi-classical method has been developed for isotropic collisions of neutral atoms or ions with electrons by Seaton (1962) and Sahal-Bréchot (1969a,b). We have applied this method for the evaluation of inelastic collisional rates. More recently, a further semi-classical method has been developed for isotropic collisions of atoms with neutral hydrogen by Anstee \& O'Mara (1991, 1995), and generalized to the evaluation of collisional elastic depolarization crosssections by Derouich et al. (2003) and Derouich (2004). 
Table 1. Observational data and results of interpretation. From left to right: observational data: date, limb distance $d_{\text {limb }}$, cosine of the heliocentric angle $\mu$, ratio of line center intensity to the neighboring continuum intensity $I_{\text {cent }} / I_{\text {cont }}$, line center linear polarization degree $p(Q / I$ of Fig. 1), theoretical continuum polarization degree $p_{\text {cont }}$ (see text); results of interpretation: height of formation of the line center above the $\tau_{5000}=1$ level $h$ (see text for its definition), microturbulent velocity $v_{\text {micro }}$, macroturbulent velocity $v_{\text {macro }}$, mircoturbulent magnetic field strength $B$, field strength in Hanle relative units ( $\Gamma$ parameter, see Sect. 3.2), where the value 1 corresponds to the maximum of sensitivity.

\begin{tabular}{ccccccccccc}
\hline \hline date & $\begin{array}{c}d_{\text {limb }} \\
\text { arcsec }\end{array}$ & $\begin{array}{c}\mu \\
\cos \theta\end{array}$ & $I_{\text {cent }} / I_{\text {cont }}$ & $\begin{array}{c}p \\
\%\end{array}$ & $\begin{array}{c}p_{\text {cont }} \\
\%\end{array}$ & $\begin{array}{c}h \\
\mathrm{~km}\end{array}$ & $\begin{array}{c}v_{\text {micro }} \\
\mathrm{km} \mathrm{s}^{-1}\end{array}$ & $\begin{array}{c}v_{\text {macro }} \\
\mathrm{km} \mathrm{s}^{-1}\end{array}$ & $\begin{array}{c}B \\
\text { Gauss }\end{array}$ & $\Gamma$ \\
\hline $2002 / 12 / 07$ & 4 & 0.091 & 0.647 & 1.244 & 0.131 & 328 & 1.80 & 1.75 & 39 & 1.72 \\
$2002 / 12 / 08$ & 4 & 0.091 & 0.642 & 1.225 & 0.131 & 328 & 1.95 & 1.75 & 42 & 1.85 \\
$2002 / 12 / 07$ & 10 & 0.144 & 0.587 & 0.959 & 0.081 & 300 & 1.90 & 1.55 & 44 & 1.93 \\
$2002 / 12 / 08$ & 10 & 0.144 & 0.587 & 0.869 & 0.081 & 300 & 2.10 & 1.75 & 50 & 2.20 \\
$2002 / 12 / 08$ & 20 & 0.203 & 0.554 & 0.829 & 0.053 & 280 & 1.95 & 1.80 & 36 & 1.57 \\
$2002 / 12 / 09$ & 20 & 0.203 & 0.558 & 0.715 & 0.053 & 280 & 1.75 & 1.65 & 46 & 2.00 \\
$2002 / 12 / 09$ & 40 & 0.286 & 0.516 & 0.522 & 0.032 & 260 & 2.05 & 1.90 & 50 & 2.20 \\
$2002 / 12 / 09$ & 80 & 0.400 & 0.503 & 0.325 & 0.018 & 239 & 1.70 & 1.90 & 54 & 2.37 \\
$2002 / 12 / 09$ & 160 & 0.553 & 0.496 & 0.199 & 0.009 & 220 & 1.65 & 2.00 & 53 & 2.33 \\
\hline
\end{tabular}

We have applied this last method for evaluating depolarizing rates due to elastic collisions with neutral hydrogen atoms (see Sect. 3).

The comparison between our observations and the polarization computed from a plane-parallel atmosphere has confirmed the existence of a depolarizing effect that can be ascribed to a turbulent weak magnetic field. In Sect. 2, we present our observations and data reduction procedure, leading to the observed intensity and polarization profiles. In Sect. 3, we describe the model for the formation of the line polarization, leading to the theoretical intensity and polarization profiles. Section 4 is devoted to the main instrumental effects that are taken into account in the theoretical calculation. The computed profiles are then compared to the observed ones, leading to the diagnostic of the turbulent magnetic field strength and of the microscopic and macroscopic velocities. The results are given and discussed in Sect. 5.

\section{Observations and data reduction}

The observations were obtained with the spectropolarimeter of the THÉMIS solar telescope (Izaña, Tenerife, Spain), on 2002, December 7-9.

\subsection{Observational procedure}

The observational procedure followed is very similar to the one described in Bommier \& Molodij (2002). The slit, 1 arcmin long and 0.5 arcsec wide, was positioned parallel to the solar limb, at the North Pole where no magnetic activity was apparently present (the data have been further averaged along the slit to increase polarimetric sensitivity). The observations were obtained for a series of distances between the slit and the limb ranging from 4 to 160 arcsec, corresponding to heliocentric angles $\theta$ from $85^{\circ}$ to $55^{\circ}$ (see Table 1). Apart from the 4 arcsec limb distance, the limb was not visible on the slit-view camera, so that the following procedure was used for fixing the limb distance: the slit was first positioned as well as possible at the intensity inflexion point as shown by a radial cut in the slitview real time image (taken in the continuum at $4307 \AA$ ). This
Table 2. Positions of the two quarter-wave plates (QWP1 and QWP2) of the analyzer, and resulting output signals after the beam splitter (last column). The first column gives the conventional name of the setting of the polarimeter. QWP1 is the first plate encountered by the Sun's light.

\begin{tabular}{crrc}
\hline \hline name & QWP1 & QWP2 & Output \\
\hline$+Q$ & $+45^{\circ}$ & $-45^{\circ}$ & $I \pm Q$ \\
$+U$ & $0^{\circ}$ & $+45^{\circ}$ & $I \pm U$ \\
$+V$ & $-45^{\circ}$ & $0^{\circ}$ & $I \pm V$ \\
$-Q$ & $+45^{\circ}$ & $+45^{\circ}$ & $I \mp Q$ \\
$-U$ & $0^{\circ}$ & $-45^{\circ}$ & $I \mp U$ \\
$-V$ & $+45^{\circ}$ & $0^{\circ}$ & $I \mp V$ \\
\hline
\end{tabular}

inflexion point was considered as the limb position. Then, the slit was moved at the desired limb distance by performing a radial displacement with the pursuit tool of the telescope.

One of the differences with respect to the 2000 campaign described in Bommier \& Molodij (2002) is that the polarization analyzer was replaced by a new version that allows beam exchange in any of the polarization Stokes parameters $(Q, U, V)$, so that we sequentially recorded the images corresponding to the 6 analyzer positions, respectively denoted as $+Q,-Q,+U,-U,+V,-V$. The corresponding quarter-wave plates positions are listed in Table 2 (the Stokes parameter are here given in the analyzer reference frame, which is rotated $45^{\circ}$ from the limb reference frame). These plates positions are the ones that give fewer fringes (Bommier \& Molodij 2002, for $U$; and Semel 2003, for $Q$ ).

As in Bommier \& Molodij (2002), the acquisition of limb images and flat field images were interleaved, in order to retrieve the same fringes positions on both series of images. Fringes can thus be eliminated from the limb images by using the flat field images (fringe displacements are probably correlated to the telescope drift, that we manually correct at each tipping). On December 7, we interleaved packages of 10 sequences of $6 \mathrm{limb}$ and flat images, twice, so that we have $20 \times 6$ limb and flat images for the two limb distances 4 and 10 arcsec observed on that day. On December 8, the packages were of 5 sequences of 6 images, interleaved four times so that 
we have $20 \times 6$ limb and flat images for the two limb positions of 4 and $10 \mathrm{arcsec}$, and interleaved two times so that we have $10 \times 6$ limb and flat images for the limb distance of 20 arcsec. On December 9, we interleaved packages of 5 sequences of $6 \mathrm{limb}$ and flat images, twice, so that we have $10 \times 6$ limb and flat images at 20, 40,80 and 160 arcsec from the solar limb. As usual, the flat field images were recorded by rapidly moving the solar image on the spectrograph slit along a random ellipse centered on disk center (or elsewhere in a quiet region if a sunspot was found in the vicinity of disk center). The integration time was $13 \mathrm{~s}$ on December 7, 8 and 11s on December 9.

Two different cameras were used to acquire the images in the two polarization states obtained after the beam splitter, $I+X$ and $I-X$, where $X$ is one of the polarization Stokes parameters. The magnification factor of the camera optics was about $1 / 2$.

\subsection{Data reduction and results}

The data reduction technique and methods are those described in Bommier \& Rayrole (2002) and Bommier \& Molodij (2002), apart from the generalized beam exchange method that has become useless with the new polarization analyzer, where the usual beam exchange is available for the three polarization Stokes parameters $Q, U$ and $V$. One of the characteristics of our method is that the data are averaged along the slit as soon as possible in the reduction process, just after line destretching and flat field correction, and before the polarization analysis (profile subtraction) which includes a correction of the difference in dispersion between the two channels.

The results of the polarization analysis are given in Fig. 1, where the Stokes parameters are now expressed in the limb reference frame, and where the dotted line is the intensity profile in arbitrary units. The intensity and polarization profiles are superimposed for all the different limb distances. The continuum polarization degree has been arbitrarily adjusted to the theoretical value, which is 0 for $U / I$. For $Q / I$, we have taken the theoretical value of the continuum polarization as given by Fluri \& Stenflo (1999), following the method described in Bommier \& Molodij (2002). The Sr I line is located at $4607.333 \AA$, and the neighbouring line is a Fe I line with wavelength $4607.651 \AA$. In $Q / I$, the polarization at the different limb distances is visible in the Sr I line (the polarization decreases when the limb distance increases), and it is clearly visible in the figure that the Fe I line "depolarizes the continuum", as shown by Stenflo et al. (1997). No significant polarization is found in $U / I$, so that no rotation of the linear polarization direction with respect to the zero magnetic field direction (the limb direction) is observed, in full agreement with the observational results of Trujillo Bueno et al. (2001) obtained for a limb distance $<1$ arcsec.

The values of the line center polarization degree $(Q / I$, now denoted as $p$ ) are given in Table 1 for the different limb distances. The theoretical continuum polarization degree, $p_{\text {cont }}$, that we have used to set the value of the observed continuum polarization is also given. It can be seen that these polarization degrees are in good agreement with those of Faurobert et al. (2001) obtained in 2000 with THÉMIS, and with the one observed in 2001 by Gandorfer (2002) with ZIMPOL II at IRSOL, Locarno. No difference is also found with our THÉMIS 2000 campaign (Bommier \& Molodij 2002), thus raising serious doubts about the possibility of a cyclic variation suggested by the difference found with the 1994 data of Stenflo et al. (1997) showing a polarization degree larger by a factor of 1.5. Further investigation is however necessary to reach firm conclusions on the possible 11-year cyclic variation of the polarization.

Table 1 also displays the ratio $I_{\text {cent }} / I_{\text {cont }}$ of the line center intensity to the neighboring continuum intensity, for the various limb distances.

\section{Model for the formation of the line polarization}

The diagnostic of the various quantities (microturbulent or macroturbulent velocity, magnetic field) is done by comparing the observed and theoretical polarization profiles. The present section describes the theoretical methods used for the interpretation of the observed profiles and for fitting the data by varying the relevant parameters. A density matrix polarized radiative transfer code has been used. Since no rotation of the linear polarization direction with respect to the limb direction is observed, a unimodal, turbulent magnetic field has been assumed, as proposed by Stenflo (1982) and Faurobert-Scholl (1993). A plane-parallel atmosphere has also been assumed.

\subsection{Polarized radiative transfer in the atomic density matrix formalism}

As already stated in the introduction, the 2-level atom approximation can be applied to the description of the Sr I $4607 \AA$ line formation. Moreover, as stated by Faurobert-Scholl (1993), partial frequency redistribution effects are not very important for this line, so that complete redistribution can be safely assumed. Under these conditions, the density matrix method for polarized radiative transfer in the presence of weak magnetic fields (Hanle effect), developed by Landi Degl'Innocenti et al. (1990), and applied by Bommier et al. (1991) to the case of a plane-parallel isothermal and isodense atmosphere permeated by a deterministic magnetic field, can be generalized to the case of a turbulent weak magnetic field in a model atmosphere.

In the density matrix formalism of Landi Degl'Innocenti et al. (1990), the quantities to be computed are the atomic density matrix elements of the upper level, expressed in the irreducible tensors basis $\left(\rho_{Q}^{K}\right)$. They obey an integral equation obtained by reporting the formal solution of the polarized radiative transfer equation into the statistical equilibrium equation for the atomic density matrix elements. On the contrary, in the "scattering method" used by Faurobert-Scholl (1993; see also Rees 1978; Faurobert-Scholl \& Frisch 1989; Faurobert-Scholl 1991), an integral equation is obtained for the generalized source function by reporting the formal solution of the statistical equilibrium equation (which is very simple for a 2-level atom) into the transfer equation. Although both methods follow different strategies, they are in principle equivalent so that no difference is expected in the results. The generalized source function and the density matrix elements are fully equivalent, apart from a normalization factor, in the complete 
redistribution approximation (Landi Degl'Innocenti et al. 1990; Landi Degl'Innocenti \& Bommier 1994; Frisch 1998).

The continuous absorption has been added to the formalism, introducing thus the additional parameter $\beta$ defined by

$\beta=\frac{k_{c}}{k_{L}}$

which is the ratio of the continuous absorption coefficient $k_{c}$ and the line absorption coefficient $k_{L}$, which is equal to the $\eta^{(0)}$ coefficient of Landi Degl'Innocenti et al. (1991, Eq. (25)), and which is also $\eta_{0}$ of Landi Degl'Innocenti et al. (1990, Eq. (13)).

To numerically solve the integral equation, the density matrix elements have to be discretized over a grid of optical depth points. As described in Bommier \& Landi Degl'Innocenti (1998), several interpolation methods for the density matrix elements between the grid points have been envisaged: linear, quadratic or cubic spline. Although the quadratic and cubic spline interpolations converge much faster and give accurate results in the isothermal and isodense atmosphere case (analytical tests are provided by the $\sqrt{\epsilon}$-law, see Landi Degl'Innocenti $\&$ Bommier 1994), they have to be abandoned due to numerical instability in the model atmosphere case, where we have then preferred the linear interpolation, the only one that is stable.

\subsection{Microturbulent magnetic field}

The magnetic field is assumed to be unimodal and microturbulent, which means that, at any optical depth, the magnetic field is constant in intensity and has an isotropic distribution of directions. Moreover, we suppose that the correlation length of the "microturbulent eddies" is infinitely small, so that the direction of the magnetic field at optical depth $\tau$ is completely uncorrelated to the direction at optical depth $\tau^{\prime}$, no matter how close the two points are in space.

The spatial average leading to the turbulent magnetic field model cannot be directly performed in the formalism developed in Landi Degl'Innocenti et al. (1990), because the magnetic kernel, which has to be angle averaged at each optical depth $\tau$, is multiplied by the density matrix element at the same optical depth $\tau$ (see Eq. (8) of that paper). A different treatment of the magnetic kernel has then to be performed (see also Trujillo Bueno and Manso Sainz 1999). The statistical equilibrium equation for the upper level density matrix element $\rho_{q}^{k}\left(J^{\prime}\right)$ at optical depth $\tau$ in a uniform magnetic field can be written as (see Landi Degl'Innocenti et al. 1990)

$$
\begin{aligned}
\left(1+\mathrm{i} \Gamma^{\prime} q\right) & {\left[1+\delta^{(k)}\left(1-\epsilon^{\prime}\right)\right] \sqrt{2 J^{\prime}+1}\left[\rho_{q}^{k}\left(J^{\prime}\right)\right]_{x y z}=} \\
& \left(1-\epsilon^{\prime}\right) \frac{B_{\mathrm{abs}}}{A} w_{J^{\prime} J}^{(k)}(-1)^{q}\left[\bar{J}_{-q}^{k}\left(v_{0}\right)\right]_{x y z} \\
& +\left(1-\epsilon^{\prime}\right) \frac{C_{J J^{\prime}}}{A} \delta_{k, 0} \delta_{q, 0} .
\end{aligned}
$$

The lower level population $\sqrt{2 J+1}\left[\rho_{0}^{0}(J)\right]_{x y z}$ that should multiply the r.h.s. of this equation, is assumed to be equal to 1 (the lower level is moreover assumed to be unpolarized). $\Gamma^{\prime}$ is the reduced magnetic field strength as defined in Eq. (59) of Landi Degl'Innocenti et al. (1990), which has to be rewritten here as

$$
\Gamma^{\prime}=\frac{\Gamma}{1+\epsilon+\delta^{(2)}}=\frac{g_{J^{\prime}} e_{0} B}{2 m c\left(A+C_{J^{\prime} J}+D^{(2)}\right)}
$$

to correct a misprint about the Landé factor $g_{J^{\prime}}$. In this equation, $\Gamma=\left(g_{J^{\prime}} e_{0} B\right) /(2 m c A)$ is the value of the magnetic field $B$ in relative units; $A, C_{J^{\prime} J}\left(\epsilon=C_{J^{\prime} J} / A\right)$ and $D^{(2)}\left(\delta^{(2)}=D^{(2)} / A\right)$ are respectively: the Einstein coefficient for spontaneous emission, the collisional de-excitation coefficient and the collisional depolarizing rate. The $\epsilon^{\prime}$ coefficient is defined as

$\epsilon^{\prime}=\frac{C_{J^{\prime} J}}{A+C_{J^{\prime} J}}$

so that

$$
\frac{1}{1+\epsilon+\delta^{(2)}}=\frac{\left(1-\epsilon^{\prime}\right)}{1+\delta^{(2)}\left(1-\epsilon^{\prime}\right)}
$$

$B_{\text {abs }}$ is the Einstein coefficient for absorption, and $w_{J^{\prime} J}^{(k)}$ is a coefficient related to the atomic polarisability, that is defined and tabulated in Landi Degl'Innocenti (1984). $\bar{J}_{-q}^{k}\left(v_{0}\right)$ is a tensor describing the incident radiation intensity and angular distribution, averaged over the absorption profile (see also Landi Degl'Innocenti 1984). The statistical equilibrium equation Eq. (2) involves tensorial quantities defined in the magnetic field reference frame $x y z$, where the $z$ axis is the magnetic field direction. Before averaging on all possible directions of the magnetic field in modelling the microturbulent field, it is necessary to rewrite this equation in the fixed atmosphere reference frame $X Y Z$, where the $Z$ axis is the vertical of the atmosphere. From

$$
\left[\rho_{q}^{k}\right]_{X Y Z}=\sum_{q^{\prime}}\left[\rho_{q^{\prime}}^{k}\right]_{x y z}\left[\mathcal{D}_{q^{\prime} q}^{(k)}\right]^{*}(\mathcal{R})
$$

and

$$
\left[\bar{J}_{q}^{k}\right]_{X Y Z}=\sum_{q^{\prime}}\left[\bar{J}_{q^{\prime}}^{k}\right]_{x y z} \mathcal{D}_{q^{\prime} q}^{(k)}(\mathcal{R})
$$

where $\mathcal{R}$ is the rotation bringing $x y z$ on $X Y Z$ and $\mathcal{D}_{q^{\prime} q}^{(k)}$ is the rotation matrix element, one gets

$$
\begin{aligned}
& {\left[1+\delta^{(k)}\left(1-\epsilon^{\prime}\right)\right] \sqrt{2 J^{\prime}+1}\left[\rho_{q}^{k}\left(J^{\prime}\right)\right]_{X Y Z}=} \\
& \quad\left(1-\epsilon^{\prime}\right) \frac{B_{\mathrm{abs}}}{A} w_{J^{\prime} J}^{(k)} \sum_{q^{\prime}} \frac{1}{1+\mathrm{i} \Gamma^{\prime} q^{\prime}} \\
& \quad \times \sum_{q^{\prime \prime}}(-1)^{q^{\prime \prime}}\left[\bar{J}_{-q^{\prime \prime}}^{k}\left(v_{0}\right)\right]_{X Y Z} \mathcal{D}_{q^{\prime} q^{\prime \prime}}^{(k)}(\mathcal{R})\left[\mathcal{D}_{q^{\prime} q}^{(k)}\right]^{*}(\mathcal{R}) \\
& \quad+\left(1-\epsilon^{\prime}\right) \frac{C_{J J^{\prime}}}{A} \delta_{k, 0} \delta_{q, 0} .
\end{aligned}
$$

This equation can be integrated over all possible directions describing the turbulent magnetic field. From the Weyl theorem (Weyl 1939; see also Brink \& Satchler 1979)

$$
\oint \frac{\mathrm{d} \boldsymbol{\Omega}}{4 \pi} \mathcal{D}_{q^{\prime} q^{\prime \prime}}^{(k)}(\mathcal{R})\left[\mathcal{D}_{q^{\prime} q}^{(k)}\right]^{*}(\mathcal{R})=\delta_{q^{\prime \prime}, q} \frac{1}{2 k+1}
$$


the statistical equilibrium equation can be written in the case of a turbulent magnetic field, as

$$
\begin{aligned}
& {\left[1+\delta^{(k)}\left(1-\epsilon^{\prime}\right)\right] \sqrt{2 J^{\prime}+1}\left[\rho_{q}^{k}\left(J^{\prime}\right)\right]_{X Y Z}=} \\
& \quad\left(1-\epsilon^{\prime}\right) \mu_{k} \frac{B_{\mathrm{abs}}}{A} w_{J^{\prime} J}^{(k)}(-1)^{q}\left[\bar{J}_{-q}^{k}\left(v_{0}\right)\right]_{X Y Z} \\
& +\left(1-\epsilon^{\prime}\right) \frac{C_{J J^{\prime}}}{A} \delta_{k, 0} \delta_{q, 0} .
\end{aligned}
$$

This equation differs from the non-magnetic polarized equation only by the presence of the $\mu_{k}$ factor, given by

$\mu_{k}=\frac{1}{2 k+1} \sum_{q^{\prime}=-k}^{k} \frac{1}{1+\mathrm{i} \Gamma^{\prime} q^{\prime}}$.

In particular

$\mu_{0}=1$

and

$\mu_{2}=\frac{1}{5}\left[1+\frac{2}{1+\left(\Gamma^{\prime}\right)^{2}}+\frac{2}{1+4\left(\Gamma^{\prime}\right)^{2}}\right]$,

which are the only ones that intervene in our calculations. The result is that the turbulent case can be easily derived from the non-magnetic case (both polarized), by simply multiplying the r.h.s. of the integral equations by the proper $\mu_{k}(k=$ $0,2)$ factor. However, as a consequence, the test provided by the analytical $\sqrt{\epsilon}$-law, as detailed by Landi Degl'Innocenti \& Bommier (1994) for arbitrary magnetic fields is no longer valid in the case of a microturbulent magnetic field.

\subsection{Atmospheric model}

The parameters entering the integral equation for the densitymatrix elements, and that are allowed to vary with depth, are the following ones: a) the temperature $T$ that determines the value of the Planck function; b) the coefficient $\epsilon^{\prime}$ describing inelastic collisions by electrons (see Eq. (4)), where $C_{J^{\prime} J}$ depends on temperature and electron density; and c) the upper level depolarizing rate $D^{(2)}$ due to elastic collisions with neutral hydrogen atoms, that depends on the temperature and neutral hydrogen density. The temperature, electron pressure and gas pressure have been taken from the Maltby et al. Quiet Sun Photospheric Reference Model (Maltby et al. 1986), extrapolated downwards beyond $-70 \mathrm{~km}$ to $-450 \mathrm{~km}$ below the $\tau_{5000}=1$ level. Above $-70 \mathrm{~km}$, this model is very similar to the Quiet Sun FAL C (Fontenla et al. 1993).

The introduction of the depth-dependence of the $\beta$ coefficient (ratio of the continuum to the line absorption coefficient defined in Eq. (1)) requires particular attention, because the argument of the integro-exponential functions $E_{n}$ entering the kernels $K$ of the integral equations (see Eqs. (56) and (61) of Landi Degl'Innocenti et al. 1990) depends on both $\beta$ and the absorption profile $\phi(x)$ where $x$ is the frequency in adimensional units $\left(x=\left(v-v_{0}\right) / \Delta v_{\mathrm{D}}\right)$ : by introducing the continuous absorption coefficient in the formalism, $E_{n}[\tau \phi(x)]$ has to be replaced by $E_{n}[\tau(\phi(x)+\beta)]$ for a constant $\beta$, and by

$E_{n}\left[\int_{0}^{\tau}\left(\phi(x)+\beta\left(\tau^{\prime}\right)\right) \mathrm{d} \tau^{\prime}\right]$ for a depth-dependent $\beta$. We have applied the trapezoidal rule to evaluate the integral that now appears as the argument of the integro-exponential function.

Finally, both the intensity of the microturbulent magnetic field and the line Doppler width $\Delta v_{\mathrm{D}}$ (which includes microturbulent velocity) have been assumed to be depth-independent. The generalization to depth-dependent fields (turbulent or not) can be easily handled by our theoretical approach, whereas the introduction of a depth-dependent Doppler width would imply a heavier modification of our codes.

\subsection{Evaluation of the grid of line center optical depths}

The numerical solution of the integral equations for the density matrix elements requires the determination of the line opacity with respect to the continuum opacity. The continuum absorption coefficient has been evaluated as in the MALIP code of Landi Degl'Innocenti (1976), i.e. by including $\mathrm{H}^{-}$boundfree, $\mathrm{H}^{-}$free-free, neutral hydrogen atom opacity, Rayleigh scattering on $\mathrm{H}$ atoms and Thompson scattering on free electrons. For the line center optical depth evaluation, atomic data have been taken from Allen (1973) and partition functions from Wittmann. The strontium abundance has been assumed to be 2.93 (in the usual logarithmic scale where the abundance of hydrogen is 12). A depth-independent microturbulent velocity field of $1 \mathrm{~km} \mathrm{~s}^{-1}$ has been introduced. LTE ionization equilibrium has been assumed by using Saha's law to determine the SrII and SrIII abundances with respect to SrI. Finally, departures from LTE in the ionization equilibrium have been simulated by applying Saha's law with a constant "radiation temperature" of $5100^{\circ} \mathrm{K}$ instead of the electron temperature provided by the atmosphere model, for depths higher than the one corresponding to $\tau_{5000}=0.1$.

The height of formation of the line center has then been determined as follows: given the grid of line center optical depths, which provides the optical depth along the vertical as a function of height from the atmosphere model, the height of formation of the line center is the one for which the optical depth along the line of sight is unity (Eddington-Barbier approximation), i.e. the one for which $\tau / \mu=1$, where $\tau$ is the line center optical depth along the vertical, and $\mu$ is the cosine of the heliocentric angle $\theta$. The heights obtained for each of the observed $\mu$ values are given in Table 1 , in $\mathrm{km}$ above the $\tau_{5000}=1$ level. The corresponding temperatures have been used to derive the value of the Doppler width $\Delta v_{\mathrm{D}}$, which also includes the microturbulent velocity (see Eq. (17)), and which is considered $\tau$-independent in our model.

\subsection{Atomic data}

\subsubsection{Radiative rates}

The Einstein spontaneous emission coefficient has been taken from NIST (2004)

$A=2.01 \times 10^{8} \mathrm{~s}^{-1}$,

leading to the oscillator strength value

$f=1.92$. 


\subsubsection{Elastic collisions: Depolarizing rates}

As already stated, the depolarizing effect due to elastic collisions (that induce depolarizing transitions between Zeeman sublevels) is mainly due to collisions with neutral hydrogen atoms. Since these collisions are isotropic, the corresponding rate for the density matrix element $\rho_{Q}^{K}$ depends only on the multipolar order $K$, so that it is denoted as $D^{(K)}$ in Landi Degl'Innocenti et al. (1990, 1991), with $D^{(0)}=0$.

A semi-classical method for computing the depolarizing rates has been derived from the formalism of Anstee \& O'Mara (1991, 1995), and is presented in Derouich et al. (2003) and Derouich (2004) with results for atomic $p$ states like the upper level of the Sr I $4607 \AA$ line. The corresponding code has been used to compute the depolarizing rate $D^{(2)}$ at each optical depth. The results can be fitted as

$D^{(2)}=1.36 \times 10^{-8} n_{\mathrm{H}}\left(\frac{T}{5000}\right)^{0.374} \mathrm{rad} \mathrm{s}^{-1}$,

where $n_{\mathrm{H}}$ is the number of neutral hydrogen atoms per cubic centimeter and $T$ is the temperature in Kelvin. This rate is in very close agreement (a few percent difference in the line formation temperature range) with the one used by Faurobert et al. (1995, Eqs. (32)-(33), 2001, Eq. (11)), obtained by a quantum chemistry method.

\subsubsection{Inelastic collisions: Transfer rates}

For the transfer rates due to inelastic collisions, that enter the $\epsilon^{\prime}$ coefficient (see Eq. (4) for definition; and Landi Degl'Innocenti et al. 1990, 1991), the main contribution is the one due to electrons. We have used the semi-classical method of Seaton (1962) and Sahal-Bréchot (1969a,b), completed with a model of momentum transfer during the collision (Bommier, to be published), to compute the excitation rates $C_{J J^{\prime}}$. The deexcitation rates $C_{J^{\prime} J}$ have been derived by applying the mircoreversibility law. The semi-classical method is estimated to be accurate to within $20 \%$. It has been found in addition that the method of Van Regemorter (1962) used by Faurobert et al. $(1995,2001)$ gives results in good agreement with the present semi-classical ones.

\subsection{Microturbulent and macroturbulent velocities}

The microturbulent and macroturbulent velocities are generally introduced to take into account non-thermal motions in the theoretical calculations of the emergent profiles. The microturbulent motions occur on scales that are small when compared to the photon mean free path, so that they constitute an additional source of line broadening besides the thermal broadening (collisional broadening has been neglected). The microturbulent velocity $v_{\text {micro }}$ has then been introduced as usual in the Doppler width $\Delta v_{\mathrm{D}}$

$\Delta v_{\mathrm{D}}=\frac{v_{0}}{c} \sqrt{\frac{2 k_{\mathrm{B}} T}{M}+v_{\text {micro }}^{2}}$,

where $v_{0}$ is the line center frequency, $c$ the speed of light, $k_{\mathrm{B}}$ the Boltzmann constant, $T$ the temperature at the height of line center formation (see the determination in Sect. 3.4), and $M$ the atomic weight taken from Allen (1973): 87.62 a.m.u. for strontium. The macroturbulent velocity corresponds to motions occuring on scales larger than the photon mean free path (Mihalas 1978). They have been taken into account by means of a convolution of the theoretical emerging profiles in each Stokes parameter with a Gaussian of the form

$\frac{1}{x \sqrt{\pi}} \exp \left\{-\left(\frac{v-v_{0}}{x \Delta v_{\mathrm{D}}}\right)^{2}\right\}$

where

$x=\frac{v_{\text {macro }}}{\sqrt{\frac{2 k_{\mathrm{B}} T}{M}+v_{\text {micro }}^{2}}}$,

$v_{\text {macro }}$ being the macroturbulent velocity (same approach as in Faurobert-Scholl et al. 2001).

\section{Introduction of the instrumental parameters in the interpretation}

The observed profiles are influenced by various instrumental effects, such as the instrumental profile due to the spectrograph non-zero slit width that introduces finite spectral resolution, diffraction limit of the telescope, scattered and stray light, ghost images in the spectrograph, etc. To compensate, we have introduced two effects in our theoretical model: the spectrograph slit width effect, which prevails by far over the diffraction limit, and the global effect of scattered, stray and ghost lights. These effects are taken into account in the evaluation of the theoretical profile to be compared with the observed one.

\subsection{Instrumental profile and spectrograph slit width effect}

The way to take into account the instrumental profile is to convolve the theoretical profile with a function that describes this instrumental effect. Instead of the instrumental profile itself, a reasonable choice is to convolve the theoretical profile by the spectral cut of the entrance slit image on the camera. This spectral cut is a stepwise function, for which we have determined the spectral width of $19 \mathrm{~m} \AA$ corresponding to the 0.5 arcsec entrance slit that we have used on THÉMIS. Performing this stepwise convolution on the theoretical profile, we have derived an increase of $1.0 \%$ of the Sr I $4607 \AA$ line center intensity, resulting thus in a rather small effect.

\subsection{Scattered, stray and ghost light effect}

The amplitude of this effect has been determined by comparing the observed profile at disk center (i.e., the mean profile of the flat field images that have been taken in the disk center vicinity), to an atlas profile (Delbouille et al. 1973). This has led us to the determination of a scattered, stray and ghost light level of $8.5 \%$ of the continuum intensity, this rate $r_{1}$ being defined as follows: in any point along the profile, it is assumed that

$I_{\mathrm{o}}(v)=I_{\mathrm{a}}(v)+I_{\mathrm{s}}$, 
$I_{0}(v)$ being the observed intensity, $I_{\mathrm{a}}(v)$ being the atlas one, and $I_{\mathrm{s}}$ the scattered light intensity assumed to be frequencyindependent and unpolarized. Both observed and atlas profiles being further normalized to their continuum level, the value of $I_{\mathrm{S}}$ in this relative unit is our rate $r_{1}$ and is determined by the comparison of the profiles at two different frequency points (one in line center and one in continuum). It has to be emphasized that $r_{1}$ is different from the line center intensity difference, both profiles being normalized to their continuum level: this other rate $r_{2}$ is related to $r_{1}$ by

$r_{2}=0.6 r_{1}$

for the Sr I $4607 \AA$ line $(0.6$ being the line depth in relative units). For our present THÉMIS observations, $r_{2}$ is then $5.1 \%$. In this determination, both observed and atlas profiles have been corrected at the Sr I $4607 \AA$ line center for the instrumental profile effect: the THÉMIS profile as described above, and the atlas profile by taking into account the instrumental profile width of $6 \mathrm{~mA}$ as displayed in Delbouille et al. (1973) (this last effect results in an increase of only $0.10 \%$ of the Sr I $4607 \AA$ A line center intensity). The parasitic light has been assumed to be unpolarized, and the corresponding level of unpolarized intensity has been added to the computed intensity before comparison with observations. It represents the global effect of the light scattered in the telescope, in the transfer optics and in the spectrograph, of the stray light, and of the ghost images. With the present rate of $8.5 \%$, this correction is not negligible at all.

\section{Results and discussion}

The results are displayed in Table 1 , in terms of $v_{\text {micro }}\left(\mathrm{km} \mathrm{s}^{-1}\right)$, $v_{\text {macro }}\left(\mathrm{km} \mathrm{s}^{-1}\right)$, and $B$ (Gauss). They have been obtained by comparing the theoretical profiles to the observed ones (modified according to the procedure outlined in the previous section to account for instrumental effects). The best fits are displayed in Fig. 2 for the data obtained on 2002 December 7 and 9 . Within a good approximation, the relative intensity profile $I / I_{\text {cont }}$ is independent of the magnetic field strength $B$, so that the velocities $v_{\text {micro }}$ and $v_{\text {macro }}$ have been firstly determined from the intensity profiles by applying a zero-field model, the magnetic field being determined in a second step from the polarization profile. The behavior of the theoretical intensity profile as a function of the velocities is as follows: the microturbulent velocity mainly determines the profile width, whereas the macroturbulent velocity mainly determines the profile height. The best fit is obtained by an iterative method which consists in first adjusting the profile width with $v_{\text {micro }}$, then the profile height with $v_{\text {macro }}$, and repeating the procedure until the difference between theoretical and observed profiles reaches its minimum. This yields a pair of values $\left(v_{\text {micro }}, v_{\text {macro }}\right)$, which is thus uniquely determined. Using this pair of values, the turbulent magnetic field strength is determined by adjusting the line center theoretical polarization profile derived from the magnetized model, to the observed one (to which continuum polarization has been subtracted), without further adjustment of the polarization profile width. Figure 3 displays the observed center-tolimb variation of the line polarization degree together with the
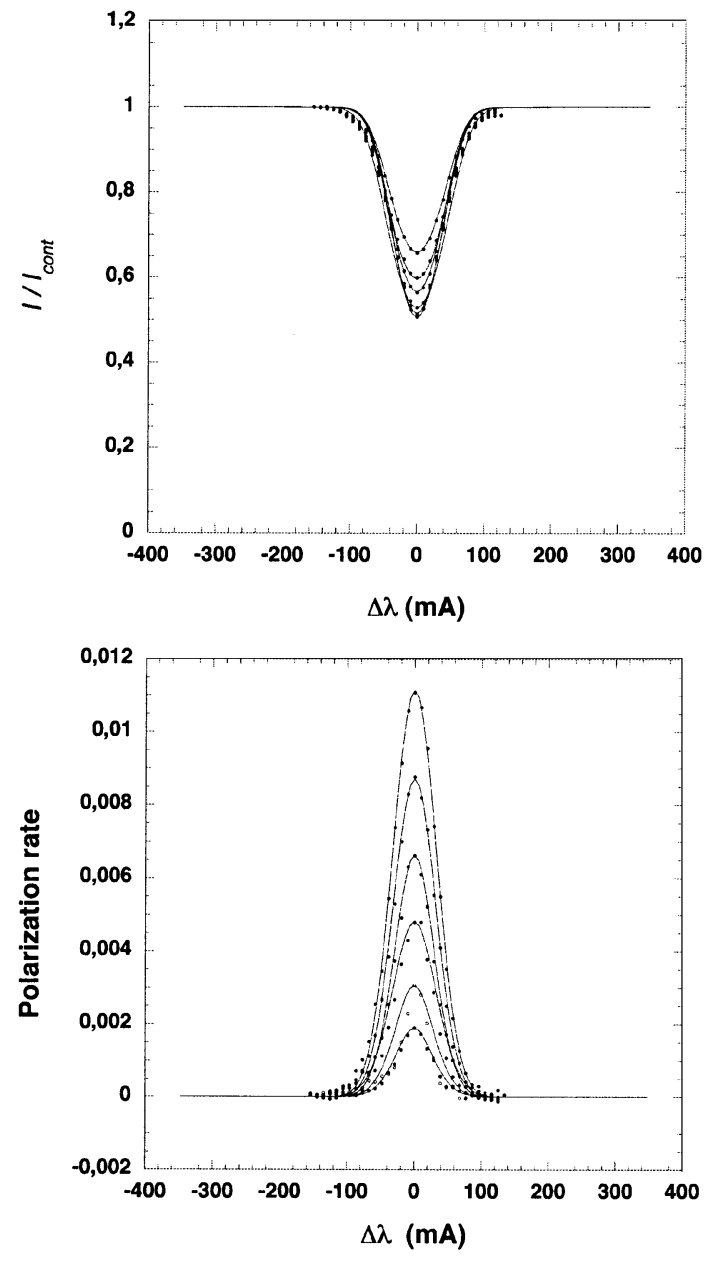

Fig. 2. Superposition of adjusted theoretical profiles (lines) and observations (dots) of 2002 December 7 and 9. The theoretical intensity profiles have been computed in a non-magnetized atmosphere and are used to determine $v_{\text {micro }}$ and $v_{\text {macro }}$. Given these values, the theoretical polarization profiles, adjusted to the observations at line center only, are used to determine the microturbulent field strength $B$ from the magnetized atmosphere model.

derived magnetic field strengths. An exponential least square fit to the observations, given by the law

$p=0.01507 \exp (-3.84 \mu)$,

is also shown.

The resulting values for the three parameters $v_{\text {micro }}, v_{\text {macro }}$, and $B$ are shown in Table 1. The average values are:

$v_{\text {micro }}=1.87 \mathrm{~km} \mathrm{~s}^{-1}$,
$v_{\text {macro }}=1.78 \mathrm{~km} \mathrm{~s}^{-1}$,
$B=46$ Gauss.

The turbulent magnetic field strength ranges from 36 to 54 Gauss, and the standard deviation over the nine observed points is 6 Gauss for the magnetic field, and 0.15 and $0.13 \mathrm{~km} \mathrm{~s}^{-1}$ for the micorturbulent and macroturbulent velocities respectively. As it can be seen in Table 1, it is found that the velocities are rather constant along the investigated depths. The measurement depth in atmosphere is however lower for $v_{\text {micro }}$, 

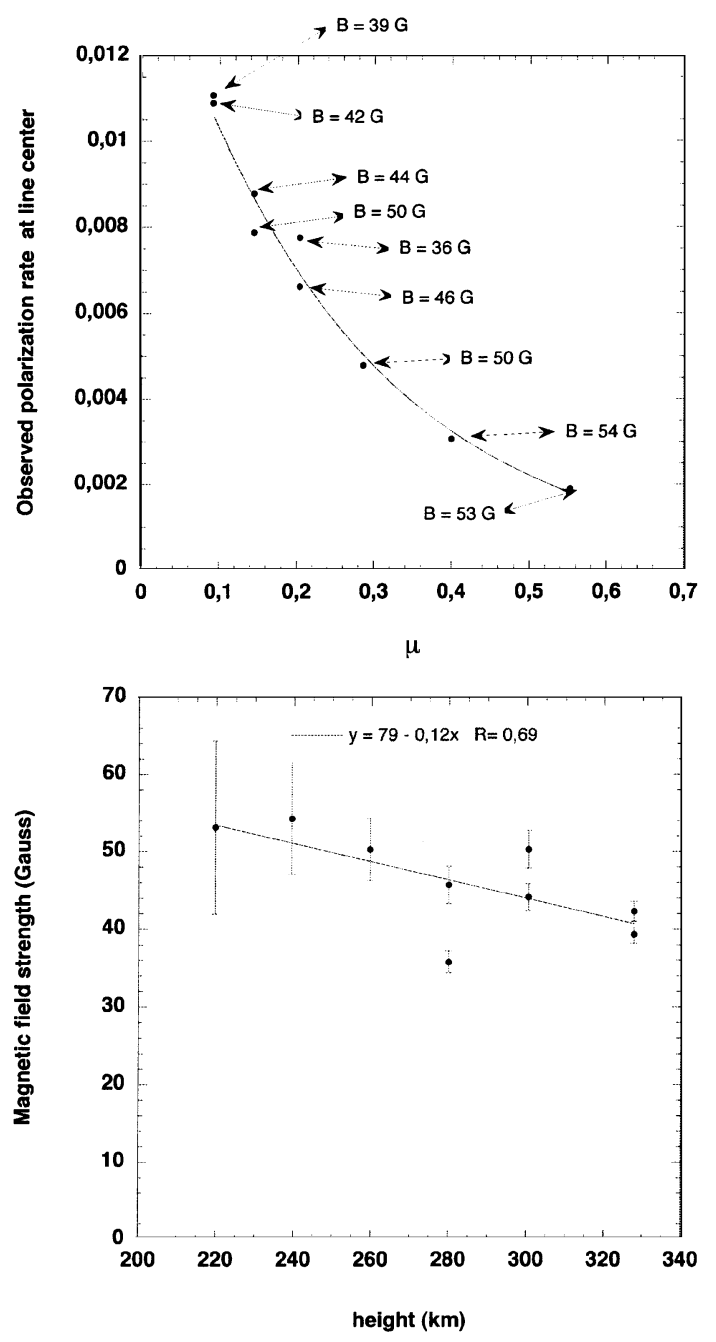

Fig. 3. Top: center-to-limb variation of the observed linear polarization (dots), with an exponential least square fit (line, see Eq. (22)), together with the values of the determined microturbulent field strength in Gauss. Bottom: microturbulent field strength in Gauss, as a function of the height above the $\tau_{5000}=1$ level in $\mathrm{km}$. This height results from our line opacity evaluation (see text). The error bars show the effect of the polarimetric inaccuracy only, the theoretical data being assumed to be sure at this stage. The line is a least square fit whose equation is provided.

mainly derived from the line width, than for $v_{\text {macro }}$, mainly derived from the line center. In addition, this $v_{\text {micro }}$ value is different from the depth-independent one introduced in the line opacity computation (see Sect. 3.4): this can be admitted because both velocities do not correspond to the same depth in the atmosphere. The variation of the magnetic field strength with height is shown in the lower part of Fig. 3. The figure shows a trend towards a decrease with height. A linear regression analysis gives a gradient of -0.12 Gauss $/ \mathrm{km}$ with a correlation coefficient $R=0.69$. The error bars on the determination of the magnetic field are assumed to be due only to the polarimetric accuracy which is estimated to be $2 \times 10^{-4}$ (see Fig. 1 and Bommier \& Molodij 2002). The inaccuracy value in terms of magnetic field strength has been obtained by the derivation of Eq. (13), ignoring the collision effects at this stage.
The present magnetic field strengths are about twice the values of 20-30 Gauss derived by Faurobert et al. (2001) from their THÉMIS observations. Faurobert et al. (2001) assume the values $v_{\text {micro }}=1 \mathrm{~km} \mathrm{~s}^{-1}$, about half the value, and $v_{\text {macro }}=2.5 \mathrm{~km} \mathrm{~s}^{-1}$, this last one being determined by adjustment of the theoretical intensity profile to the observed one. When we use Faurobert's values of $v_{\text {micro }}$ and $v_{\text {macro }}$ on our observational data, we find the adjustement unsatisfying, but we then derive the turbulent field strength in the range 1428 Gauss (average value 23 Gauss) from our model and our observations, in good agreement with the field strength of 2030 Gauss determined by Faurobert et al. (2001), which shows that both theoretical methods are in good agreement.

The value $v_{\text {micro }}=1 \mathrm{~km} \mathrm{~s}^{-1}$ that has been used by Faurobert et al. (2001) has been taken from Fontenla et al. (1991). The microturbulent velocity value of Fontenla et al. (1991) is the result of a semi-empirical determination, and, as the authors point out, is of minor importance in their model, because it is used for introducing the turbulent pressure that does not play a very important role.

We think that a proper adjustment of the theoretical profile to the observed one requires two free parameters, one mainly sensitive to the width and one mainly sensitive to the depth, as $v_{\text {micro }}$ and $v_{\text {macro }}$ are respectively. With only one free parameter, as done by Faurobert et al. (2001), the adjustment cannot be fully achieved.

Shchukina \& Trujillo Bueno (2003) have considered the FAL-C model including its given height-dependent microturbulent velocity, and they have also concluded that, by varying only $v_{\text {macro }}$, it is impossible to fit both line width and height. Shchukina \& Trujillo Bueno find a difference of a factor of 2 in the derived microturbulent field strength, according to the performed adjustment: the field strength is found to be about two times higher when the adjustment is performed on the line height.

\subsection{Effect of the accuracy of the depolarizing collision rates}

We have analyzed the effect of a possible inaccuracy of the depolarizing collision rates on the retrieved value of the turbulent magnetic field. From a numerical evaluation, we have obtained that, as the depolarizing collision rates are given within $20 \%$ or less inaccuracy by the semi-classical method (Derouich et al. 2003; Derouich 2004), the induced inaccuracy on the magnetic field strength is only 6\% or less ( $\operatorname{Sr}$ I $4607 \AA$ case). This is due to the fact that the Hanle effect is characterized by the factor $\Gamma^{\prime}$ defined in Eq. (3). For a fixed $\Gamma^{\prime}$, we have, by differentiation

$$
\frac{\delta B}{B}=-\frac{D^{(2)}}{A+C_{J^{\prime} J}+D^{(2)}} \frac{\delta D^{(2)}}{D^{(2)}},
$$

which explains our findings since $A \simeq D^{(2)}$ and $C \ll A$. It can then be concluded that the magnetic field strength is well determined, even if some uncertainty remains on the depolarizing collision rates. 


\section{Conclusion}

Similarly to previous authors, we come to a conclusion about the existence of a microturbulent magnetic field, as shown by the fact that no rotation of the polarization direction with respect to the limb direction is observed (see Fig. 1), and by the fact that the observed polarization is considerably lower than what is expected from plane-parallel, realistic models of the solar atmosphere. The main criticism that can be made to these observations is that the spatial resolution is poor, because the data have been averaged along the slit which was one arcmin long. To increase the spatial resolution without decreasing too much the polarimetric sensitivity probably requires further instrumental progress.

From three observed quantities, which are the line width, height and center polarization, we derive by adjustment three physical quantities: $v_{\text {micro }}, v_{\text {macro }}$ and the turbulent field strength $B$. In particular, the adjustment of the line width is essential.

Theoretical modelling of the SrI $4607 \AA$ line can be improved only by a full non-LTE treatment of the Sr II/Sr I ionization equilibrium (multi-level radiative transfer), and by applying multi-dimensional radiative transfer to magneto-convective simulations of the solar atmosphere, as done by Shchukina \& Trujillo Bueno (2003) and Trujillo Bueno et al. (2004). These improvements are welcome since they will contribute to evaluate more realistically the polarization of the Sr I $4607 \AA$ line without the need to introduce ad-hoc parameters such as $v_{\text {micro }}$ and $v_{\text {macro }}$. We think, however, that the results obtained in the present paper on the value of the turbulent field will not be much affected by these more sophisticated investigations: indeed, Trujillo Bueno et al. (2004) derive a magnetic field strength of 60 Gauss, in the volume-filling field case, for $\mu \geq$ 0.2 , which is in very good agreement with our values of 50 , 54 and 53 Gauss obtained in this $\mu$ range. This validates our method.

For any Hanle magnetic field determination, the polarization rate in the absence of a magnetic field has to be evaluated, in order to retrieve the magnetic depolarization. In the present analysis, the zero-field polarization degree is derived from a theoretical model, so that the present determination can be qualified as a model-dependent one. Two kinds of model-independent techniques have indeed been proposed with promising objectives: the differential Hanle effect, where the magnetic field is derived from the ratio of two lines of different magnetic sensitivity (see analysis in Stenflo et al. 1998; Berdyugina \& Fluri 2004). However, in this case, the model independence is rigorously obtained only if the two lines are formed in the same conditions. The second model-independent technique concerns lines showing far coherent wings that are insensitive to the magnetic field, unlike the line core. For the moment, such a feature has been found in one line only, Ca I $4227 \AA$. The technique of Hanle histograms based on this feature can be found in Bianda et al. (1998), but the field determination is statistical. In an analysis like the present one, the magnetic field strength is determined together with the height of formation of the line resulting from the model, thus providing some information on the height dependence of the field strength, which is of great interest for now understanding the solar atmosphere.

Acknowledgements. The authors are indebted to M. Faurobert for clarifying advice, in particular on the macroturbulent velocity effect.

\section{References}

Allen, C. W. 1973, Astrophysical Quantities, 3rd Edition (Univ. of London, the Athlone Press)

Anstee, S. D., \& O'Mara, B. J. 1991, MNRAS, 253, 549

Anstee, S. D., \& O'Mara, B. J. 1995, MNRAS, 276, 859

Berdyugina, S. V., \& Fluri, D. M. 2004, A\&A, 417, 775

Bianda, M., Solanki, S. K., \& Stenflo, J. O. 1998, A\&A, 331, 760

Bommier, V., \& Landi Degl'Innocenti, E. 1998, in THÉMIS International Workshop on Radiative Transfer and Inversion Codes, Tenerife (Spain), ed. C. Briand, February 9-11, Paris Observatory Publ., 17

Bommier, V., \& Molodij, G. 2002, A\&A, 381, 241

Bommier, V., \& Rayrole, J. 2002, A\&A, 381, 227

Bommier, V., Landi Degl'Innocenti, E., \& Sahal-Bréchot, S. 1991, A\&A, 244, 383

Brink, D. M., \& Satchler, G. R. 1979, Angular Momentum (Oxford: Clarendon)

Delbouille, L., Roland, G., \& Neuven, L. 1973, Photometric atlas of the solar spectrum from $3000 \AA$ to $10000 \AA$, Institut d'Astrophysique, Liège University (Belgium)

Derouich, M. 2004, Thèse de doctorat, Université Paris VII Denis Diderot (France)

Derouich, M., Sahal-Bréchot, S., Barklem, P. S., \& O’Mara, B. J. 2003, A\&A, 404, 763

Faurobert-Scholl, M. 1991, A\&A, 246, 469

Faurobert-Scholl, M. 1993, A\&A, 268, 765

Faurobert-Scholl, M., \& Frisch, H. 1989, A\&A, 219, 338

Faurobert-Scholl, M., Feautrier, N., Machefert, F., Petrovay, K., \& Spielfiedel, A. 1995, A\&A, 298, 289

Faurobert, M., Arnaud, J., Vigneau, J., \& Frisch, H. 2001, A\&A, 378, 627

Frisch, H. 1998, A\&A, 338, 683

Fluri, D., \& Stenflo, J. O. 1999, A\&A, 341, 902

Fontenla, J. M., Avrett, E. H., \& Loeser, R. 1991, ApJ, 377, 712

Fontenla, J. M., Avrett, E. H., \& Loeser, R. 1993, ApJ, 406, 319

Gandorfer, A. 2000, The Second Solar Spectrum. A high spectral resolution polarimetric survey of scattering polarization at the solar limb in graphical representation, Vol. I: $4625 \AA$ to $6995 \AA$, Hochschulverlag AG an der ETH Zürich

Gandorfer, A. 2002, The Second Solar Spectrum. A high spectral resolution polarimetric survey of scattering polarization at the solar limb in graphical representation, Vol. II: $3910 \AA$ to $4630 \AA$, Hochschulverlag AG an der ETH Zürich

Landi Degl'Innocenti, E. 1976, A\&AS, 25, 379

Landi Degl'Innocenti, E. 1984, Sol. Phys., 91, 1

Landi Degl'Innocenti, E., \& Bommier, V. 1994, A\&A, 284, 865

Landi Degl'Innocenti, E., Bommier, V., \& Sahal-Bréchot, S. 1990, A\&A, 235, 459

Landi Degl'Innocenti, E., Bommier, V., \& Sahal-Bréchot, S. 1991, A\&A, 244, 391

Maltby, P., Avrett, E. H., Carlsson, M., et al. 1986, ApJ, 306, 284

Mihalas, D. 1978, Stellar Atmospheres (2nd edition) (San Francisco: Freeman)

NIST 2003, http://physics.nist.gov/cgi-bin/ AtData/main_asd

Rees, D. E. 1978, Publ. Astron. Soc. Jpn, 30, 455 
Sahal-Bréchot, S. 1969a, A\&A, 1, 91

Sahal-Bréchot, S. 1969b, A\&A, 2, 322

Seaton, M. J. 1962, Proc. Phys. Soc., 79(512), 1105

Semel, M. 2003, A\&A, 401, 1, and private communication

Shchukina, N., \& Trujillo Bueno, J. 2002, in Solar Polarization 3,

Porc. meeting held in Tenerife, September 30-October 4, ed. J.

Trujillo Bueno, \& J. Sánchez Almeida, ASP Conf. Ser., 307, 336

Stenflo, J. O. 1982, Sol. Phys., 80, 209

Stenflo, J. O. 2002, in Astrophysical Spectropolarimetry, Proc. XII

Canary islands Winter School of Astrophysics, Puerto de la Cruz,

Tenerife, Spain, November 13-24, 2000, ed. J. Trujillo Bueno, F.

Moreno-Insertis, \& F. Sánchez (Cambridge: University Press), 55

Stenflo, J. O., Twerenbold, D., \& Harvey, J. W. 1983a, A\&AS, 52, 161
Stenflo, J. O., Twerenbold, D., Harvey, J. W., \& Brault, J. W. 1983b, A\&AS, 54, 505

Stenflo, J. O., Bianda, M., Keller, C. U., \& Solanki, S. K. 1997, A\&A, 322,985

Stenflo, J. O., Keller, C. U., \& Gandorfer, A. 1998, A\&A, 329, 319

Trujillo Bueno, J., \& Manso Sainz, R. 1999, ApJ, 516, 436

Trujillo Bueno, J., Collados, M., Paletou, F., \& Molodij, G. 2001, in Advanced Solar Polarimetry, Proc. 20th NSO/Sac Peak Summer Workshop, ed. M. Sigwarth, ASP Conf. Ser., 236, 141

Trujillo Bueno, J., Shchukina, N., \& Asensio Ramos, A. 2004, Nature, 430, 326

Van Regemorter, H. 1962, ApJ, 136, 906

Weyl, H. 1939, Classical Groups (Princeton University Press) 